\title{
Gene regulation by sense-antisense overlap of polyadenylation signals
}

\author{
RUI GU, ${ }^{1}$ ZUO ZHANG, ${ }^{2}$ JOSHUA N. DECERBO, and GORDON G. CARMICHAEL \\ Department of Genetics and Developmental Biology, University of Connecticut Stem Cell Institute, University of Connecticut Health Center, \\ Farmington, Connecticut 06030-3301, USA
}

\begin{abstract}
We show here that expression of genes from convergent transcription units can be regulated by the formation of doublestranded RNA (dsRNA) in the region of overlapping polyadenylation signals. The model system employed is the mouse polyomavirus. The early and late genes of polyomavirus are transcribed from opposite strands of the circular viral genome. At early times after infection, the early genes are expressed predominantly. Late gene expression increases dramatically upon the onset of DNA replication, when a major defect in polyadenylation of the late primary transcripts generates multigenomic RNAs that are precursors to the mature late mRNAs. Embedded in these late pre-mRNAs are sequences complementary to the early RNAs that act to down-regulate early gene expression via A-to-I editing of dsRNAs. In this system, the defective polyadenylation, and consequently the production of multigenomic late RNAs, depends on the context, and perhaps also, on the A-to-I editing of the poly(A) signal that overlaps the $3^{\prime}$-end of early transcripts.
\end{abstract}

Keywords: RNA editing; antisense RNA; dsRNA; polyadenylation

\section{INTRODUCTION}

The polyomavirus genome is small, $\sim 5 \mathrm{~kb}$, and contains two divergently expressed transcription units whose expression is temporally regulated during the viral life cycle (Fig. 1A). The early genes encoding large, middle, and small $\mathrm{T}$ antigens are expressed by alternative splicing of early-strand transcripts. These proteins help to regulate the viral life cycle and are involved in virus-induced oncogenic cell transformation (Benjamin 2001; Wang and Carmichael 2004). The virion structural proteins VP1, VP2, and VP3 are similarly produced by alternative splicing of late-strand transcripts. During the polyoma life cycle, there is striking temporal regulation of viral gene expression (Kamen et al. 1974, 1980a,b; Griffin and Fried 1975). Immediately after infection, RNA from the early transcription unit (E-RNA)

Present addresses: ${ }^{1}$ Division of Infectious Disease, Department of Health, 120 New Scotland Avenue, Albany, NY 12208, USA; ${ }^{2}$ Genetically Engineered Models Department, Merck Research Laboratories, 126 East Lincoln Avenue, Rahway, NJ 07065-0512, USA.

Reprint requests to: Gordon G. Carmichael, Department of Genetics and Developmental Biology, University of Connecticut Stem Cell Institute, University of Connecticut Health Center, 263 Farmington Avenue, Farmington, CT 06030-3301, USA; e-mail: carmichael@nso2.uchc.edu; fax: (860) 679-8345.

Article published online ahead of print. Article and publication date are at http://www.rnajournal.org/cgi/doi/10.1261/rna.1608909. begins to accumulate; however, RNA from the late transcription unit (L-RNA) accumulates more slowly. At $12 \mathrm{~h}$ after infection, the early-late RNA ratio is about four to one (Kamen et al. 1974; Piper 1979; Hyde-DeRuyscher and Carmichael 1988), and in the presence of DNA replication inhibitors, the ratio can be 10 to one, or higher. At $12-15 \mathrm{~h}$ post-infection, viral DNA replication commences and L-RNA begins to accumulate rapidly, while E-RNA accumulates at a slower rate. In fact, the absolute amount of E-RNA in the cell is similar at $12 \mathrm{~h}$ and $24 \mathrm{~h}$ post-infection. Thus, there is a dramatic change in the relative abundances of E- and L-RNA; by $24 \mathrm{~h}$ post-infection, the early to late RNA ratio is as low as one to 50 (Kamen et al. 1974; Piper 1979; Hyde-DeRuyscher and Carmichael 1988). This earlylate "switch" is dependent on viral DNA replication; if replication is inhibited, E-RNA accumulates to abnormally high levels with minimal accumulation of L-RNA (Cogen 1978; Heiser and Eckhart 1982; Kamen et al. 1982; Farmerie and Folk 1984; Hyde-DeRuyscher and Carmichael 1988). This temporally regulated switch is not controlled mainly at the level of transcription initiation; rather, it results from changes in late-strand transcription elongation and/or RNA stability (Hyde-DeRuyscher and Carmichael 1988; HydeDeRuyscher and Carmichael 1990; Liu and Carmichael 1993; Liu et al. 1994). We reported earlier that late RNA accumulation is regulated post-transcriptionally (Liu and 
A
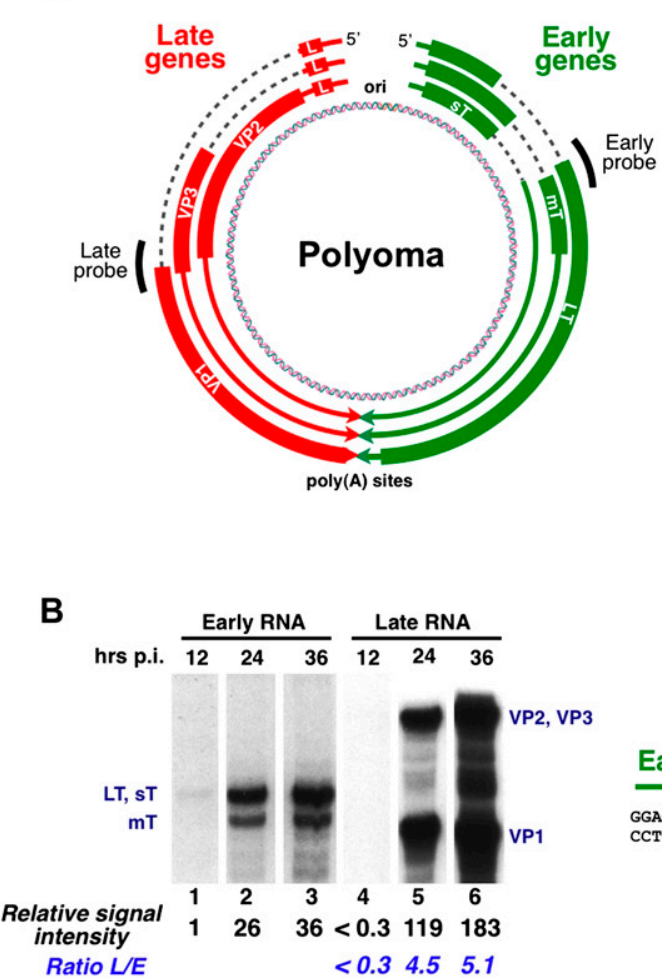

C

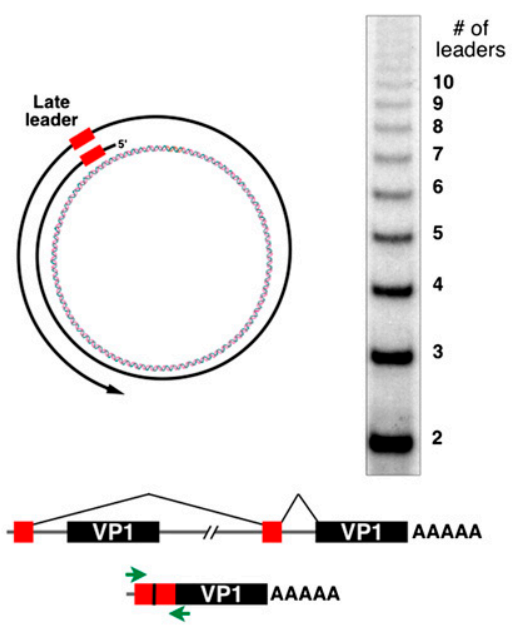

D

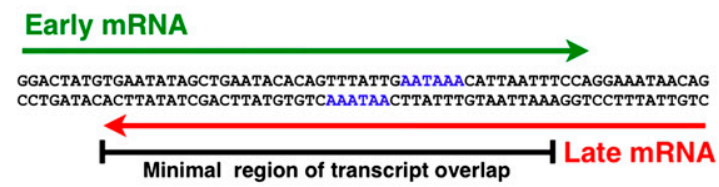

FIGURE 1. (A) The polyomavirus genome. Early and late genes are transcribed from opposite strands of the genome. (Thick green lines) Coding regions for the early genes (large, middle, and small T antigens); (thick orange lines) coding regions for the late genes (virus capsid proteins VP1, VP2, and VP3). (B) An example of the early-to-late switch. Mouse NIH3T3 cells were infected with wild-type virus and RNase protection assays performed at various times after infection, using probes specific for early-strand and late-strand mRNAs (Hyde-DeRuyscher and Carmichael 1988), which are also indicated in A. Quantitation of bands was done using ImageJ software, with the values for the bands of early RNAs at $12 \mathrm{~h}$ after infection arbitrarily set to 1 , and all comparisons made to this value. $(C)$ Processing of late pre-mRNAs. Late pre-mRNAs contain tandem copies of the noncoding late leader exon. These exons are efficiently spliced to one another, leading to the further stabilization and processing of late mRNAs (Hyde-DeRuyscher and Carmichael 1988, 1990; Liu and Carmichael 1993). (Bottom) RT-PCR can be used to reveal leader-to-leader splicing. (Right) RT-PCR assay showing that most late mRNAs have multiple tandem leader exons at their $5^{\prime}$-ends. Shown for convenience here are mRNAs with two or more leader exons. $(D)$ Early-strand and late-strand transcripts overlap. While the mRNA 3 '-end overlap is 45 bp, primary transcripts likely overlap by a significantly greater extent, leading to the potential for dsRNA formation in the nucleus.

Carmichael 1993), while early gene expression is regulated by sense-antisense RNA interactions, which result in extensive adenosine-to-inosine (A-to-I) editing of earlystrand RNA molecules at late times in infection (Liu et al. 1994; Kumar and Carmichael 1997). In this study, we examine a major unsolved question regarding the polyoma life cycle: what is the trigger that initiates the early-to-late switch? Results show that the switch is intimately associated with the overlap, and perhaps also on the A-to-I editing, of the polyadenylation signals for the early and late mRNAs. More broadly, these results suggest that poly(A) site overlap can provide a novel mode of gene regulation.

\section{RESULTS}

A typical example of polyoma gene regulation is shown by RNase protection assays using probes specific for spliced early and late mRNAs (Fig. 1B). Early-strand expression is observed at all times during infection and modestly increases after the onset of viral DNA replication (12-18 h post-infection [p.i.]). In contrast, late transcripts are only barely observable at early times (Fig. 1B, lane 4), but their levels rise rapidly at late times (Fig. 1B, lanes 5,6). We, and others, have reported that the early-to-late switch is characterized by a change in the efficiency of usage of the viral late polyadenylation signal. At early times, polyadenylation and transcription termination are efficient on the late strand; these transcripts are relatively unstable in the nucleus and accumulate to only low levels (Hyde-DeRuyscher and Carmichael 1988, 1990; Liu and Carmichael 1993; Liu et al. 1994). At late times, however, polyadenylation and transcription termination become inefficient, leading to multigenomic primary transcripts (Acheson et al. 1971; Acheson 1976, 1978; Birg et al. 1977; Treisman 1980; Treisman and Kamen 1981). Late pre-mRNAs contain at their $5^{\prime}$-ends a short noncoding "late leader" exon (Fig. 1C). Splicing of leader exons to one another in multigenomic transcripts removes the read-through sequences 
(Treisman and Kamen 1981). This stabilizes the pre-mRNAs and allows further splicing events that generate functional late mRNAs (Hyde-DeRuyscher and Carmichael 1990). As shown by an RT-PCR assay (Fig. 1C), mature late mRNAs contain tandem copies of the leader exon at their $5^{\prime}$-ends. In addition to their role as precursors for late mRNA processing, the multigenomic late-strand pre-mRNAs may serve another purpose in regulation: they contain antisense sequences that can base pair with early transcripts. We have previously shown that these antisense RNAs induce extensive A-to-I editing of early-strand transcripts and thereby down-regulate early-strand gene expression (Kumar and Carmichael 1997).

While the early-to-late switch is connected to viral DNA replication in an as-yet-unknown way, it also involves a transition from efficient to inefficient transcription termination. In the process of identifying the cis-acting sequences that control this switch (summarized in Gu et al. 2006), we concluded that the key regulatory element(s) most likely lay in the region of the late polyadenylation signal. We noted that the early and late polyadenylation signals, in fact, overlap one another (Fig. 1D). Could this overlap itself contribute to the early-to-late switch? Overlapping transcripts produced by convergent transcription units have the potential to anneal, generating RNA duplexes in the nucleus. Furthermore, these duplexes might serve as substrates for the editing enzymes ADAR1 and/or ADAR2 (Bass 2002). If this occurred in the case of polyoma, it would readily offer a plausible molecular basis for the switch (Fig. 2). According to this hypothesis, at early times, although both strands are transcribed, the relative instability of latestrand transcripts and their inefficient splicing lead to only low-level accumulation of late mRNAs (Fig. 2A). The onset of DNA replication, however, might promote the annealing of the overlapping regions. In our working model, such duplex formation, perhaps associated with the editing of the late $\operatorname{poly}(\mathrm{A})$ signal, would prevent normal processing and lead to transcriptional read-through. Leader-to-leader splicing would allow such read-through transcripts to be processed productively. Transcript overlap and possibly editing of the poly(A) signal for early-strand transcripts would also lead to transcription read-through (Fig. 2B). However, in this case, the elongated transcripts would not be processed properly, leading to nuclear decay and hence to the down-regulation of early genes.

This new model leads to three important predictions that can be addressed experimentally. First, overlapping poly(A) sites of convergent transcripts should be able to anneal to form duplex structures in vivo. If this happened, these duplexes might serve as substrates for ADAR editing. Thus, we sought evidence for A-to-I editing in the region of poly(A) site overlap. In previous work, we sequenced RTPCR products of polyoma RNAs isolated at late times in infection and identified a number of clones with edited polyadenylation signals ( $\mathrm{Gu}$ et al. 2006). Figure 3A presents evidence that at late times in infection late $\operatorname{poly}(\mathrm{A})$ region editing occurs. RT-PCR was carried out with nuclear RNA isolated at late times from polyomavirus-infected cells, and the product digested with a restriction enzyme, NdeI, whose cleavage site is near the poly(A) signals and which is abolished by editing of adenosine residues within it. Results show that a significant fraction of the product is resistant to NdeI cleavage, consistent with promiscuous editing in this region. In time-course experiments, we have observed that resistance not only to NdeI, but also to other restriction enzyme cleavage sites throughout the early region, appears only at late times in infection (Fig. 3B,C).

Cloning and DNA sequencing of cDNAs from the viral overlapping regions also indicate that editing occurs, but has not allowed us to estimate the precise frequency of editing. For reasons we do not yet understand, in different cloning experiments, we have observed variable frequencies of edited sequences, even when using the same starting material. However, some direct evidence has been obtained. Nuclear RNA was isolated $60 \mathrm{~h}$ after virus infection of NIH3T6 cells and used for RT-PCR amplification using primers that spanned the early and late polyadenylation 
A
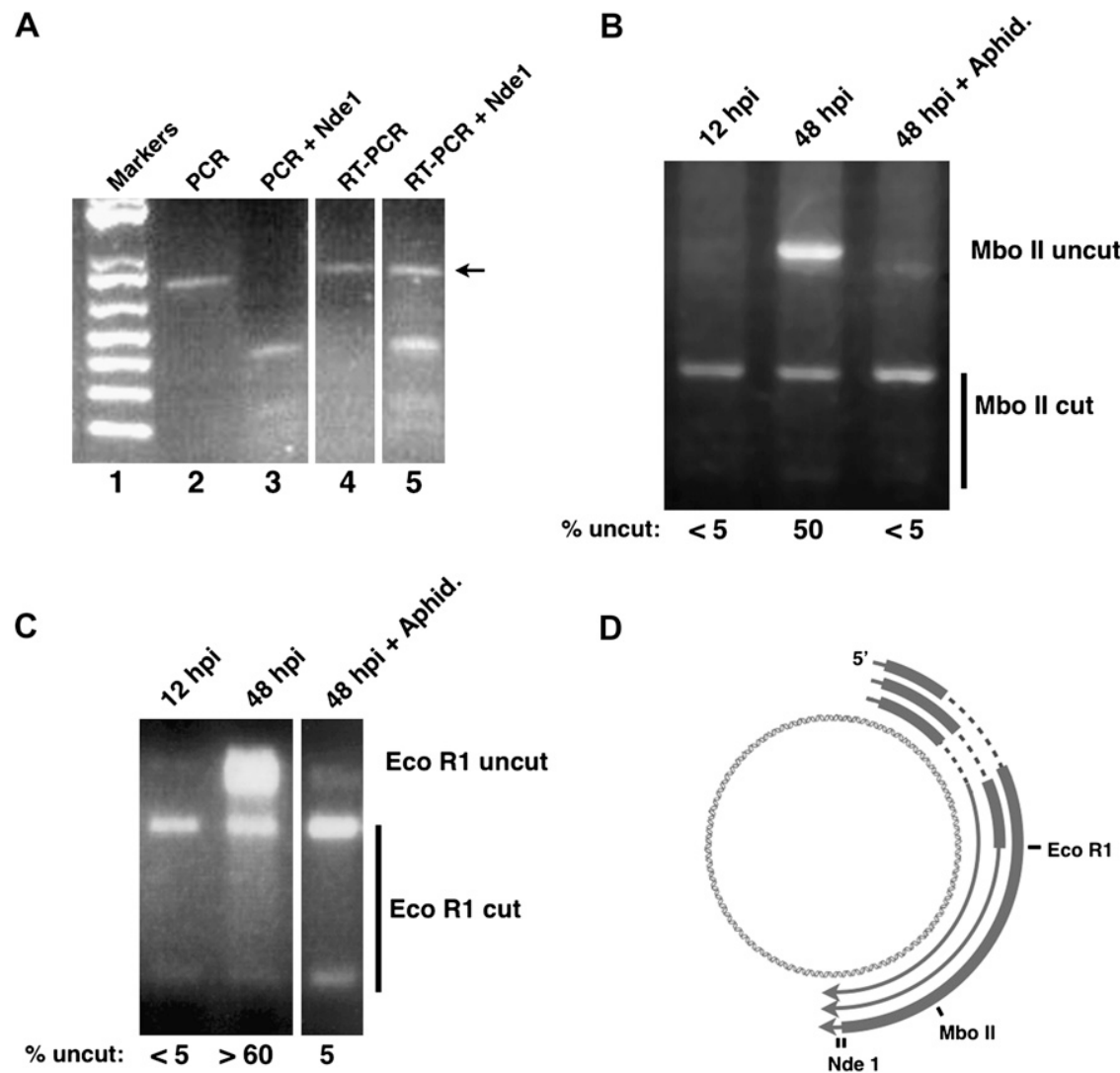

D

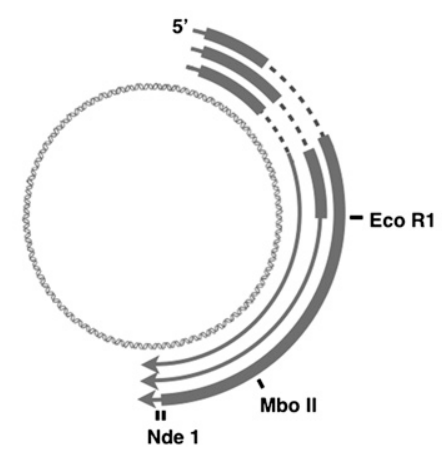

FIGURE 3. Evidence for editing in the region of the late poly(A) signal. $(A)$ RT-PCR was performed on nuclear RNA isolated $48 \mathrm{~h}$ after infection of NIH3T3 cells with wild-type polyomavirus using primers spanning the NdeI sites illustrated in $D$. These sites lie just upstream of the early poly(A) site and $21 \mathrm{nt}$ downstream from the late poly(A) site. Evidence for editing is observed by resistance to cleavage by NdeI site 21 . In this experiment, $\sim 30 \%$ of the RT-PCR product was resistant to NdeI (arrow). (B) Time-course analysis of restriction enzyme resistance of RT-PCR products. RNA tested included that from $12 \mathrm{~h}$ post-infection (hpi), 48 hpi and 48 hpi in the presence of aphidicolin to inhibit DNA replication and the early-late switch (Liu and Carmichael 1993). (C) As in A, but RT-PCR was with primers spanning the MboII site shown in $D$.

signals. Following TOPO-TA cloning, individual clones were picked and sequenced. In this experiment, seven of 44 randomly selected clones showed evidence of editing in the 3 '-region of early-strand RNAs (Fig. 4A). Since editing requires duplex formation, editing of early RNAs also indicates that the complementary late transcripts must also be edited in this region. Figure $4 \mathrm{~B}$ shows a sequence from one clone from the late poly $(\mathrm{A})$ site region in which an A-to-G transition (indicative of A-to-I editing) is found in the first position of the late-strand AAUAAA hexanucleotide.

Another hallmark of edited RNAs is that they contain inosines. We therefore sought evidence of this in the late poly(A) site region. $\mathrm{p} 54^{\mathrm{nrb}}$ is a nuclear protein that binds to promiscuously edited and inosine-containing RNAs with high affinity (Zhang and Carmichael 2001). We immunoprecipitated nuclear extracts with anti-p54 ${ }^{\text {nrb }}$ or anti hnRNP $\mathrm{U}$ (control) antibodies and examined the precipitated RNA by RT-PCR for the presence of specific sequences (Fig. 4C). Anti-p54 ${ }^{\mathrm{nrb}}$ antibodies strongly enriched for sequences in the poly(A) region and in the polyoma early region (which were previously shown to be highly edited) (Kumar and Carmichael 1997). However, they did not enrich for viral late region coding sequences or sequences from a cellular transcript (Cox-2) that is not subject to editing and that does not appear to be bound by $\mathrm{p} 54^{\mathrm{nrb}}$. In contrast, anti-hnRNP U antibodies immunoprecipitated the Cox-2 3'-UTR region, as expected (Cok et al. 2003), but not any polyomaviral sequences. Similar results were seen in experiments comparing RNAs immunoprecipitated with anti-hnRNP C to those immunoprecipitated with anti- p54 ${ }^{\text {nrb }}$ (data not shown).

A second and important prediction of our model is that ADAR knockdown should influence the early-to-late switch. In the absence of editing, late-strand transcripts might terminate efficiently, thus preventing the accumulation of read-through products, thus interfering with the viral life cycle. Figure 4D shows that this appears to be the case. Using siRNA, we succeeded in reducing ADAR1 expression fourfold in NIH3T3 cells (Fig. 4D, lanes 1-3). When cells with reduced ADAR1 expression were infected with wild-type virus and RNA was examined $48 \mathrm{~h}$ later, there was a clear defect in the early-to-late switch (Fig. 4D, cf. lane 5 and lane 7). In control experiments, we found no evidence that ADAR1 affects viral DNA replication (data not shown). Although some residual ADAR1 activity remained, the late-to-early RNA ratio in siRNA-treated cells was reduced by a factor of 20 . No effects on the early-to-late switch have been seen in control siRNA-treated cells where ADAR1 levels are not reduced (data not shown). Thus, viral infection is sensitive to ADAR1 levels in cells.

The third, and, we believe, the most critical prediction of our model, is that viruses that retain all known genetic regulatory signals but that do not allow transcript overlap should show a defect in the early-to-late switch. To test this prediction, we constructed viral genomes that do not allow overlapping transcription of the poly (A) signals. The parent construct is virus $\mathrm{YZ}-3$, in which a synthetic poly(A) signal replaces the normal viral late poly(A) signal (Fig. 5). This virus grows like wild type and displays a normal earlyto-late switch ( $\mathrm{Gu}$ et al. 2006). Importantly, this result demonstrates that the wild-type sequence of the polyoma late poly(A) signal is not itself essential for virus growth. 
A

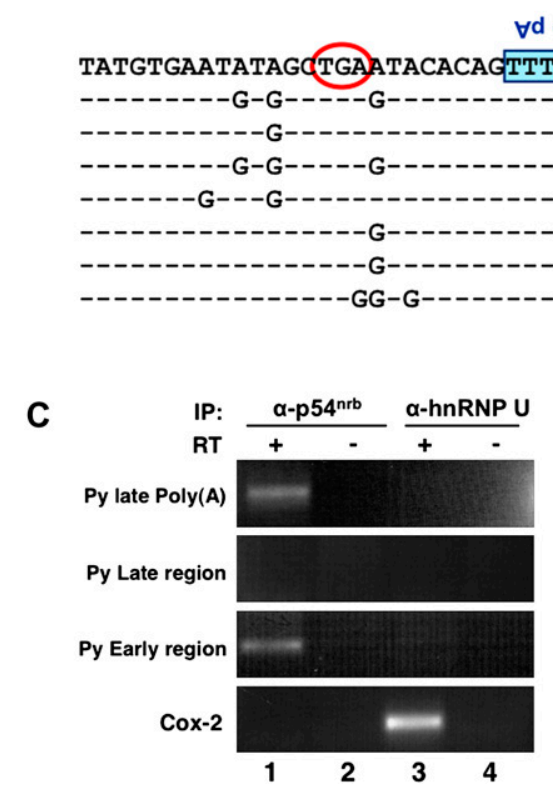

B
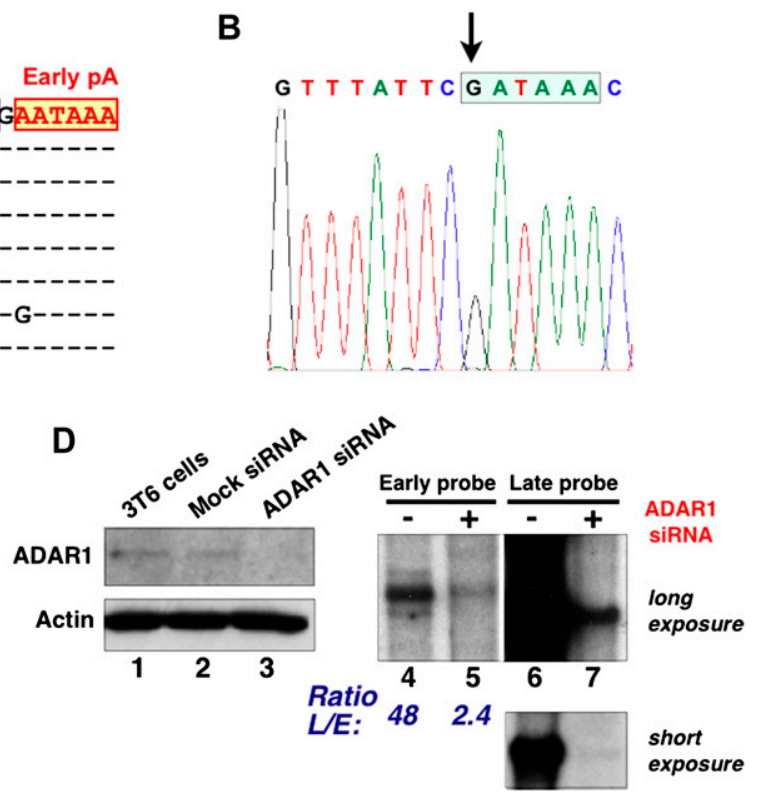

FIGURE 4. (A) Demonstration that the region of overlap between early and late polyadenylation signals leads to A-to-I editing. Nuclear RNA was isolated $60 \mathrm{~h}$ after virus infection of NIH3T6 cells. One microgram was used for RT-PCR amplification using primers that spanned the early and late polyadenylation signals. Following TOPO-TA cloning, individual clones were picked and sequenced. Seven of 44 randomly selected clones showed evidence of editing in this region. The early and late poly(A) signals are indicated by the yellow and blue boxes, respectively, and the stop codon for large T antigen is circled. (Top) The sequence of the early coding region; (below) sequences are identical except for the bases noted. Note that since editing requires duplex formation with the antisense strand, editing of early RNAs also indicates that the complementary late transcript must also have been edited in this region. $(B)$ Editing of the late poly(A) signal. NdeI-resistant RT-PCR products generated as in Figure 3B were cloned and subjected to DNA sequence analysis. In the sequence shown here, there is an A-to-G transition (arrow) at the first position of the late polyadenylation AAUAAA hexanucleotide element (boxed), indicative of editing at this position. (C) Nuclear extracts were prepared $48 \mathrm{~h}$ after infection. Immunoprecipitation was carried out with anti-p54 ${ }^{\text {nrb }}$ or anti-hnRNP $U$ antibodies, followed by RT-PCR for specific viral sequences (for $\mathrm{p} 54^{\mathrm{nrb}}$ ) or for a known target of hnRNP U in the Cox-2 $3^{\prime}$-UTR (Cok et al. 2003). Note that $\mathrm{p} 54^{\mathrm{nrb}}$ associates with the late poly(A) region and the early region, consistent with editing, but not with the viral late region, which is not edited. (D, lanes 1-3) ADAR1 siRNA was used to reduce ADAR1 expression in NIH3T3 cells. Western blotting showed about a fourfold reduction in protein levels. (Lanes 4-7) Cells treated with mock siRNA or with ADAR1 siRNA were infected with wild-type polyomavirus. Forty-eight hours later, RNase protection assays showed a 20 -fold difference in late/early RNA levels.

Between the unique BglII and XhoI sites engineered into YZ-3, we next inserted a cassette containing the bovine growth hormone and human $\beta$-globin poly(A) signals, on opposite strands. This construct is called Space-75, to denote 75 nucleotides (nt) separating the AAUAAA sequences of the oppositely oriented poly(A) signals (Fig. 5). Between the two new poly(A) signals in Space-75 we placed a unique NotI restriction enzyme cleavage site, which was subsequently used for the introduction of a variety of additional sequences to serve as spacer elements between the poly(A) signals. Analysis of a representative group of these is shown in Figure 6, A and B. Recombinant viral genomes were transfected into NIH3T6 cells, and 48 h later, RNAs were extracted and examined by RNase protection assays for defects in the early-to-late switch. Figure 6A, lanes 1 and 6 , shows that Space-75 has a normal early-to-late switch. Although its poly(A) signals do not themselves overlap, it is quite likely that the primary transcripts do, as transcription typically proceeds hundreds of nucleotides past the poly(A) signal before cleavage/polyadenylation takes place (Chao et al. 1999; Kim and Martinson 2003). Thus, the results for Space-75 are consistent with primary transcript 3 '-end overlap, leading to dsRNA formation, editing, and an early-to-late switch. Remarkably, however, longer spacer inserts strongly affect the switch. Construct Space-271 shows a partial defect in the early-to-late switch (Fig. 6A, for Space-75, cf. lanes 2,7 and lanes 1,6), while all longer inserts lead to severe defects in the induction of late gene expression and essentially lack an early-to-late switch. Note that a defect in late RNA accumulation is also associated with a defect in splicing of the VP1 mRNA compared to VP2 mRNA (Fig. 6A, lanes 8-10), as we have observed before (Hyde-DeRuyscher and Carmichael 1988, 1990). Numerous other constructs with spacer inserts longer than 300 base pairs (bp) all show the same defect (data not shown). Thus, the outcome depends on the length rather than the sequence of the spacer insert. It should be noted that in each of these constructs, all known viral regulatory sequences are intact, including promoters, splicing signals, and binding sites for viral proteins. 


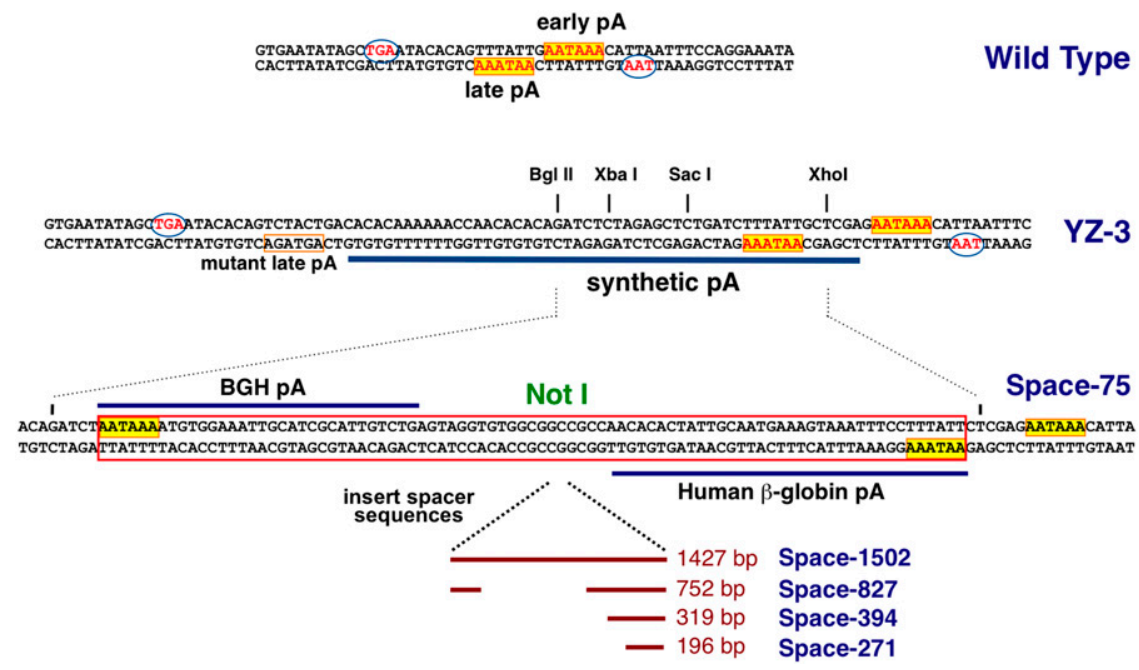

FIGURE 5. (A) Mutant viruses with altered late polyadenylation signals. (Boxes) AAUAAA elements; (circles) translation stop codons for viral proteins. Mutant YZ-3 was described previously and grows like wild type, with a normal early-to-late switch (Gu et al. 2006). Mutant Space-75 was made by replacing the BglII-XhoI segment of YZ-3 with a fragment containing the bovine growth hormone (BGH) or human $\beta$-globin poly(A) signals, separated by $75 \mathrm{bp}$ and with a unique NotI site for the insertion of additional spacer sequences, indicated at the bottom.

Figure $6 \mathrm{~B}$ shows that the constructs with defective earlyto-late switches also fail to accumulate late-strand RNA sequences downstream from the poly(A) region, again consistent with less-efficient transcription read-through. Additional control experiments with individually mutated poly(A) signals in some of these constructs (Fig. 7) further supported these conclusions. Of note, the results shown in Figure 6 did not rule out the possibility that the spacer inserts simply separate two essential cis-elements important for late polyadenylation, rather than eliminate the potential for transcript overlap. Thus, two constructs (Space-1502-E1 and Space-1502-E2) were made in which sequences immediately downstream from the late poly(A) signal were repositioned to near their original locations relative to the late region. These constructs were also found to be defective in the early-to-late switch (Fig. 7C).

Finally, we have begun to ask whether gene regulation by poly(A) site overlap may occur not just in polyomavirus infection, but also in mammalian cellular genes. The ABHD1 and Sec1 2 genes on chromosome 2 are convergently expressed and overlap by $3 \mathrm{nt}$ at their $3^{\prime}$-ends (Fig. 8A). After using siRNA to reduce ADAR1 expression in HeLa cells (Fig. 8B), we observed a dramatic change in the expression from these genes, while untreated cells and cells treated with a control siRNA exhibit a different expression pattern (Fig. 8C,D).

\section{DISCUSSION}

We have shown here that dsRNA formation and/or editing of a poly(A) signal not only reduces polyadenylation efficiency, but also leads to transcription read-through in the polyomavirus model system. The genetic regulation afforded by transcriptional read-through can be either positive or negative, depending on the nature of sequences downstream from the poly(A) sites. In polyomavirus, read-through of the late poly $(\mathrm{A})$ site leads to up-regulation of late mRNA levels because the read-through transcripts allow new and productive splicing events to occur. This is reminiscent of the regulation of the production of mRNAs encoding the membrane-associated or secreted forms of the IgM protein, where alternative splicing is masked by efficient polyadenylation or transcription termination (Bruce et al. 2003). The difference in the polyoma system is that regulation is achieved by the RNA structure or editing of signals rather than by modulation of polyadenylation or splicing factors. Conversely, the early $\operatorname{poly}(\mathrm{A})$ signal is similarly affected, but here the read-through primary transcripts are inefficiently processed. We speculate that these RNAs are either rapidly degraded or retained at the site of transcription in the nucleus (Custodio et al. 1999; Maquat and Carmichael 2001).

While we favor a model in which poly(A) site editing regulates gene expression, our data do not rule out the alternative possibility that, while editing occurs in the poly(A) site region, it is not the direct cause of transcription read-through. Rather, it remains conceivable that duplex formation occurring after RNA polymerase II passes the AAUAAA hexanucleotide and before the deposition of the polyadenylation factor CPSF may interfere with normal $3^{\prime}$-end formation. Editing would then be a consequence but not a direct cause of transcription read-through. In this scenario, dsRNA formation rather than editing would be the driving force for the switch. However, the strong effect of ADAR1 activity on viral growth (Fig. 4D) is most consistent with a direct role for editing. We also note that recent work has suggested an alternative mechanism that may contribute to the early-late switch. Kaneko et al. (2007) reported that the $\mathrm{p} 54^{\mathrm{nrb}} / \mathrm{PSF}$ complex recruits the exonuclease XRN2 to facilitate $3^{\prime}$-end processing and transcription termination. Thus, RNA editing of either the early or late strand of polyoma transcripts might lead to local titration of the $\mathrm{p} 54^{\mathrm{nrb}} / \mathrm{PSF}$ complex and thus enhance poly(A) site read-through, whether or not the polyadenylation site itself is edited.

To date, ADAR editing has been found to be involved in generation of protein diversity (Bass 2002), RNA nuclear retention (Zhang and Carmichael 2001), and perhaps heterochromatic gene silencing (Wang et al. 2005). Our 
A
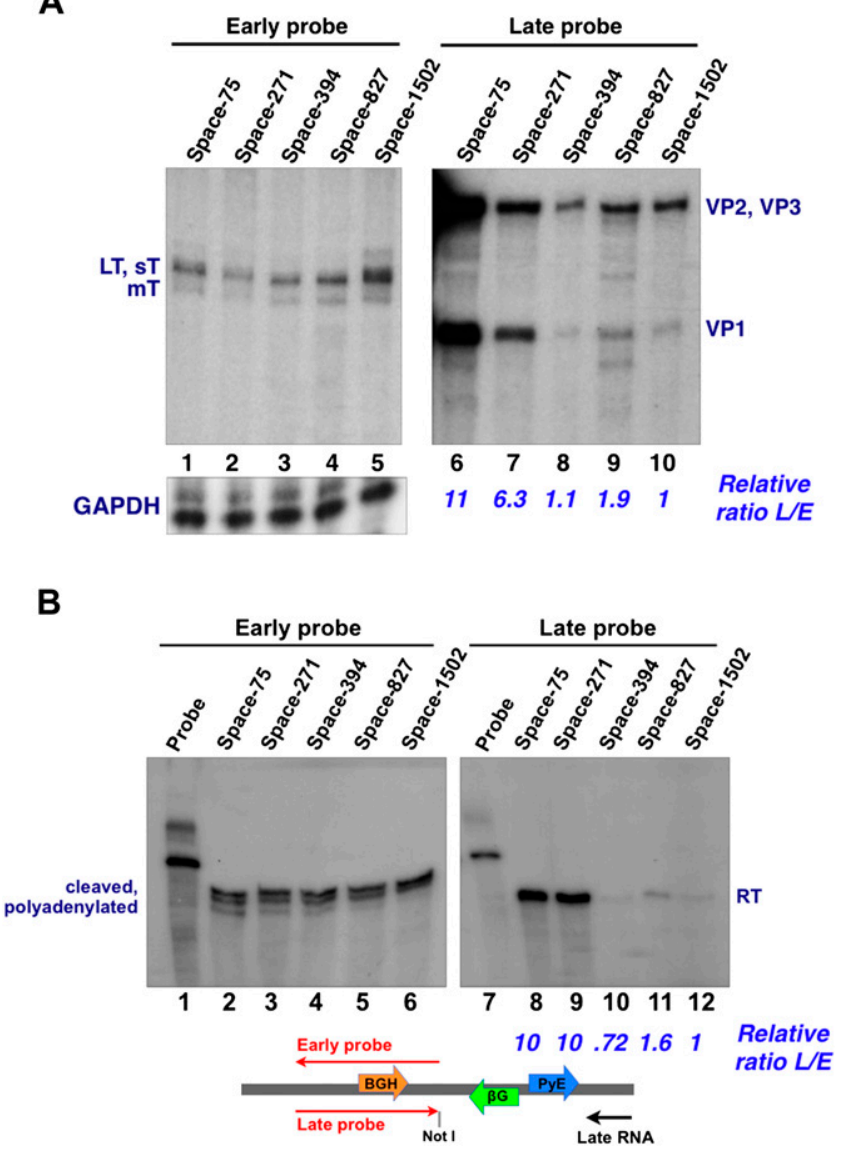

FIGURE 6. (A) RNase protection assays of mRNA from transfected cells show that Space-75 has a normal early-to-late switch, but that as additional spacer sequences are inserted, the switch is strongly affected. Mutant names represent the clones indicated in Figure 5 and indicate the spacing between opposing poly(A) signals. For the spacing constructs shown, insertion of fragments in either orientation had the same effects (data not shown), indicating that distance but not sequence is important for the effects. Equal loading of samples was confirmed using a probe for GAPDH as an internal control, and quantitation was as described in Figure 1B. The L/E signal ratio for Space-1502 was arbitrarily set to 1 , and all other ratios were compared to it. (B) Constructs with defective early-to-late switches also show lower levels of primary transcripts downstream from the late poly(A) site. Equal loading was confirmed using GAPDH as in $A$, and relative $\mathrm{L} / \mathrm{E}$ ratios were also determined as in $A$.

results here point to yet a new role for ADAR in gene regulation. It is quite possible that this new regulation is influenced by the expression levels of ADAR1 and ADAR2, which likely differ in a tissue-specific or developmentally controlled manner.

Could transcriptional interference from the oppositely oriented early and late transcription units be of importance in polyomavirus gene regulation? We believe that this is an unlikely possibility. Like the genomes of lower eukaryotes such as yeast, polyoma has a densely packed genome with transcription occurring in both directions. It has been reported (Prescott and Proudfoot 2002) that in budding yeast transcriptional collision between convergent genes can reduce gene expression, supporting the notion that transcription termination between such genes is important for maintaining normal expression. In yeast, altering the expression from one promoter in a convergent pair of genes sometimes affects the steady-state levels of mRNAs from the gene on the opposing strand (Peterson and Myers 1993; Puig et al. 1999). However, in the yeast system, the effects are mirrored by changes in nuclear run-on patterns, while we did not see any evidence of this in the polyoma system (Hyde-DeRuyscher and Carmichael 1988).

Polyomavirus depends on the host for much of its expression. The question thus naturally arises as to whether gene regulation by poly(A) site editing also occurs for cellular genes. There are hundreds, perhaps thousands, of human genes with overlapping 3 '-ends, and the conservation of such $3^{\prime}$-overlaps is significantly higher among vertebrates than that of overlapping $5^{\prime}$-ends (Sun et al. 2005). This conservation led to the suggestion that $3^{\prime}$-overlaps might influence gene regulation (Sun et al. 2005). Since both the level of ADAR and the amount of editing are higher in the brain (Bass 2002), it is tempting to speculate that such regulation may be more prevalent in this organ. The results presented in Figure 8 are consistent with such a role of ADAR editing in the regulation of gene expression.

Finally, since extensive dsRNA is produced during polyomavirus infection, the possibility exists that some of this might be processed to generate siRNAs or miRNAs that could influence the viral life cycle. Expression of miRNAs have been reported in other polyomaviruses (Sullivan et al. 2005; Seo et al. 2008, 2009), and evidence has been provided that these miRNAs can regulate their viral life cycles. It has recently been reported that murine polyomavirus also encodes a miRNA that can cleave early RNA transcripts (Sullivan et al. 2009). This miRNA, however, was found to be dispensable for infection, raising the possibility that the polyomavirus miRNA may play a subtle role in experimental infection or a yet unappreciated role in the natural host (Sullivan et al. 2009). In agreement with these results, we have in repeated attempts (data not shown) been unable to detect any evidence of miRNA or endogenous siRNA regulation in our system.

\section{MATERIALS AND METHODS}

\section{Cell culture, infection, and transfection}

NIH3T6 and NIH3T3 cells were maintained and infected with mouse polyomavirus strain 59RA as described (Benjamin 1970; Ruley and Fried 1983) at 40\%-50\% confluence. Transfections were carried out using reagent TransIT-3T3 (Mirus) following the manufacturer's protocol.

\section{siRNA gene silencing}

To knock down ADAR1 in NIH3T3 cells, an siRNA expression vector was constructed for the target sequence $5^{\prime}$-AACAAGATC 
A

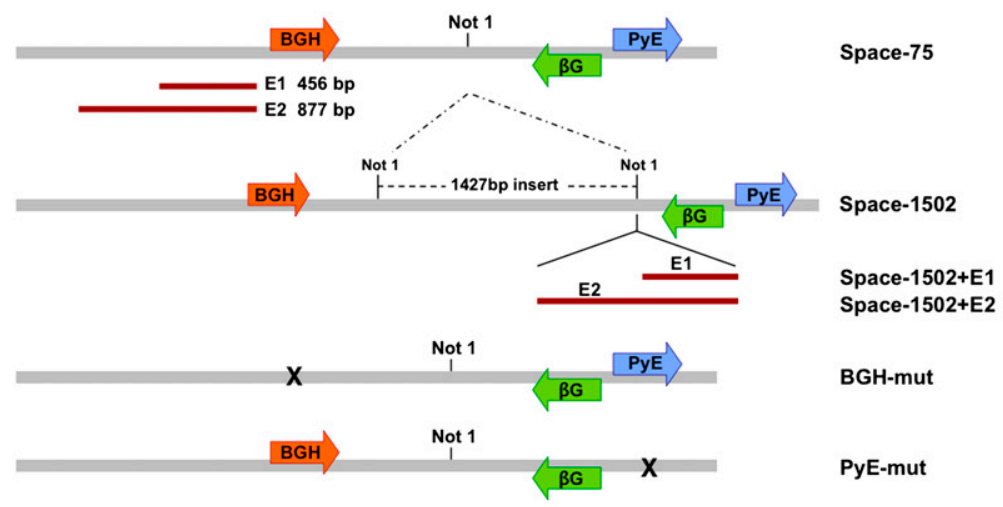

B

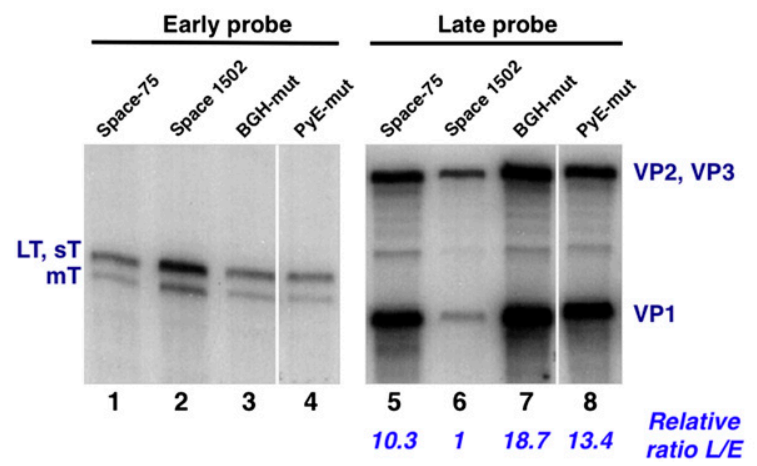

C

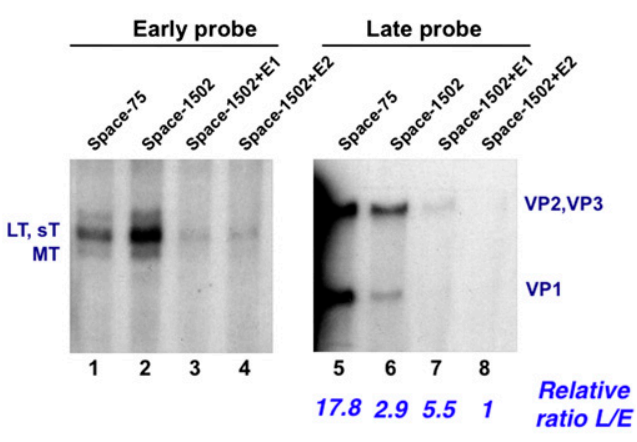

FIGURE 7. (A) Schematic of additional constructs. While the results shown in Figure 6 show that increasing the spacing between polyadenylation signals alters the E/L switch, it remained possible that the essential spacing was not between poly(A) signals themselves, but between elements flanking them. To rule this out, sequences in the early region that lie just downstream from the wild-type late poly(A) signal (E1 and E2) were inserted into construct Space-1502, as shown. To examine the contribution of tandem poly(A) signals that exist on the early strand of construct Space-75, either the bovine growth hormone (BGH) (AAUAAA to AGGAAA) or the polyoma early poly(A) signals (AAUAAA to AGUAGA) were individually mutated. $(B)$ RNase protection assays and quantitations as in Figure 6. Note that the BGH-mut and PyE-mut constructs have a normal early-to-late switch. $(C)$ RNase protection assays and quantitations as in $B$, showing that sequences downstream from the late poly(A) signal cannot rescue the early-to-late switch defect of construct Space-1502.

AGGAGGATTGGT-3' using Ambion's pSilencer 2.0 according to the manufacturer's directions, and transfected into 3T3 cells using Fugene 6 (Roche). To knock down ADAR1 in HeLa cells, duplex siRNAs for the target sequence 5'-UGGCAGUGACGGUGUC UGC-3' were synthesized by Dharmacon and transfected into HeLa cells using TransIT-TKO (Mirus) following the manufacturer's manual.

\section{RNA isolation and analysis}

Total and nuclear RNA were harvested from cells using guanidinium isothiocyanate (GTCN). RNase protection assays were carried out using universally labeled RNA probes made by in vitro transcription using T3 or T7 RNA polymerase in the presence of $\left[\alpha-{ }^{32} \mathrm{P}\right] \mathrm{UTP}$. For RT-PCR, RNA was subjected to DNase I (Roche) digestion to remove contaminating DNA. Firststrand cDNA was synthesized using the SuperScript III first strand cDNA synthesis kit from Invitrogen, following the manufacturer's instructions. Subsequent PCR was carried out using Titanium Taq from BD Biosciences.

\section{Cloning}

Mutant YZ-3 was made as described previously (Gu et al. 2006). Subsequently, DNA oligonucleotides containing the BGH poly(A) site and the $\beta$-globin poly(A) site were used to replace the BglIIXhoI fragment of YZ-3, to produce mutant Space-75. Subsequently, fragments of different length, derived from pBR322, were inserted into the NotI site of Space-75 to produce Space-271, -394, -827, and -1502. Mutant viruses PyE-mut and BGH-mut were constructed from Space-75 using oligo-directed mutagenesis.

\section{RNP immunoprecipitation}

NIH3T6 cells transfected with plasmids carrying mutant polyomaviruses or infected with wild-type (WT) polyomavirus were collected at designated time points using RNA precipitation buffer (50 mM Tris- $\mathrm{HCl}$ at $\mathrm{pH} 7.4,300 \mathrm{mM} \mathrm{NaCl}, 0.05 \% \mathrm{NP}-40,1 \mathrm{mM}$ PMSF, $1 \mathrm{mg} / \mathrm{mL}$ Aprotinin, $1 \mathrm{mg} / \mathrm{mL}$ Leupeptin, and $200 \mathrm{nM}$ vanadyl ribonucleoside complexes) and sonicated for $30 \mathrm{sec}$, twice with a 2-min interval on ice. Supernatants of the sonication 
A

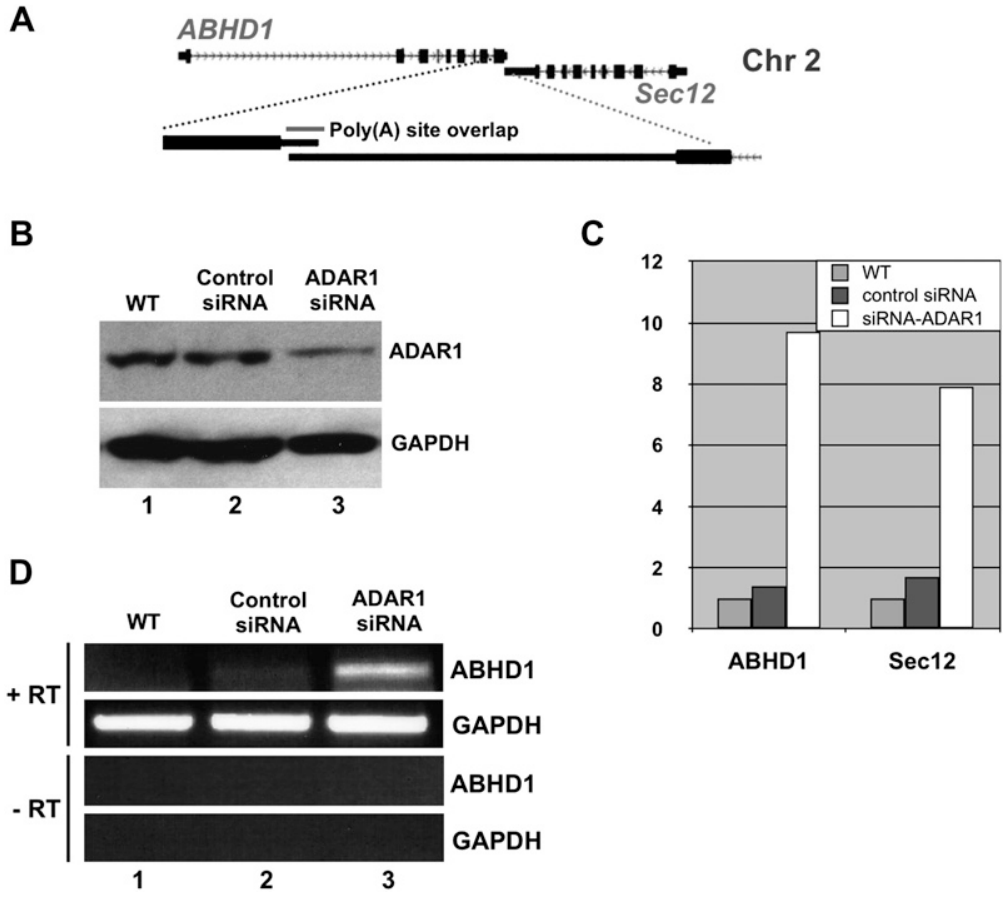

FIGURE 8. Expression from cellular genes sharing overlapping polyadenylation signals is affected by ADAR editing. (A) In the human genome, ABHD1 and Sec 12 on chromosome 2 are expressed from opposite strands of the chromosome. Their AAUAAA polyadenylation signals overlap by $3 \mathrm{nt}$, generating potential transcript overlap very similar to that seen in polyomavirus. $(B)$ Using siRNA, ADAR1 levels in HeLa cells were reduced by about threefold, compared with control and mock siRNA (against GFP) transfected samples. (C) Quantitative real-time RT-PCR shows that in the ADAR1 knockdown cells, the mRNA levels of mature ABHD1 and Sec12 both increase, compared to their levels in untreated cells or cells treated with siRNA against GFP. The results shown are representative of several independent experiments, with almost identical results. $(D)$ RT-PCR supports the results from $C$. thanks are given to Asis Das for comments on the manuscript. This work was supported by grant CA045382 from the NIH.

Received February 18, 2009; accepted March 26, 2009.

\section{REFERENCES}

Acheson, N. 1976. Transcription during productive infection with polyoma virus and simian virus 40. Cell 8: 1-12.

Acheson, N.H. 1978. Polyoma giant RNAs contain tandem repeats of the nucleotide sequence of the entire viral genome. Proc. Natl. Acad. Sci. 75: 4754-4758.

Acheson, N., Buetti, E., Scherrer, K., and Weil, R. 1971. Transcription of the polyoma virus genome: Synthesis and cleavage of giant late polyoma-specific RNA. Proc. Natl. Acad. Sci. 68: 2231-2235.

Bass, B.L. 2002. RNA editing by adenosine deaminases that act on RNA. Annu. Rev. Biochem. 71: 817-846.

Benjamin, T.L. 1970. Host range mutants of polyoma virus. Proc. Natl. Acad. Sci. 67: 394-399.

Benjamin, T.L. 2001. Polyoma virus: Old findings and new challenges. Virology 289: 167173.

Birg, F., Favaloro, J., and Kamen, R. 1977. Analysis of polyoma viral nuclear RNA by miniblot hybridization. Proc. Natl. Acad. Sci. 74: 3138-3142.

Bruce, S.R., Dingle, R.W., and Peterson, M.L. 2003. B-cell and plasma-cell splicing differences: A potential role in regulated immunoglobulin RNA processing. RNA 9: 1264-1273.

products were pre-cleared with protein $\mathrm{A} / \mathrm{G}$ plus agarose beads (Santa Cruz) for $30 \mathrm{~min}$ at $4^{\circ} \mathrm{C}$, incubated with anti-p5 $4^{\mathrm{nrb}}$ antibodies, or anti-hnRNP $U$ antibodies, protein $\mathrm{A} / \mathrm{G}$ plus agarose beads, and $0.1 \mathrm{mg} / \mathrm{mL}$ yeast RNA for $1.5 \mathrm{~h}$ at $4^{\circ} \mathrm{C}$. After incubation, the beads were washed with RNA precipitation buffer four times, $5 \mathrm{~min}$ each, and eluted with extraction buffer $(100 \mathrm{mM}$ Tris- $\mathrm{HCl}$ at $\mathrm{pH} 6.8,4 \%$ SDS, $12 \% \beta$-mercaptoethanol, $20 \%$ glycerol) at room temperature for $10 \mathrm{~min}$. RNA was extracted from the eluates with Trizol (Invitrogen) and subjected to DNase I (Roche) and Proteinase K (Invitrogen) digestions. After subsequent Trizol extraction and ethanol precipitation, RNA was used for RT-PCR analysis.

\section{SUPPLEMENTAL MATERIAL}

Supplemental material can be found at http://www.rnajournal.org.

\section{ACKNOWLEDGMENTS}

We are grateful to all of the members of the laboratory, past and present, who have contributed insights and comments throughout this work. In particular, we acknowledge the contributions of D. Batt, Z. Cai, L.-L. Chen, R. Hyde-DeRuyscher, M. Kumar, Z. Liu, and Y. Zhang, and the technical assistance of K. Morris. Special
Chao, L.C., Jamil, A., Kim, S.J., Huang, L., and Martinson, H.G. 1999. Assembly of the cleavage and polyadenylation apparatus requires about 10 seconds in vivo and is faster for strong than for weak poly(A) sites. Mol. Cell. Biol. 19: 5588-5600.

Cogen, B. 1978. Virus-specific early RNA in 3T6 cells infected by a tsA mutant of polyoma virus. Virology 85: 222-230.

Cok, S.J., Acton, S.J., and Morrison, A.R. 2003. The proximal region of the 3 '-untranslated region of cyclooxygenase-2 is recognized by a multimeric protein complex containing HuR, TIA-1, TIAR, and the heterogeneous nuclear ribonucleoprotein U. J. Biol. Chem. 278: 36157-36162.

Custodio, N., Carmo-Fonseca, M., Geraghty, F., Pereira, H.S., Grosveld, F., and Antoniou, M. 1999. Inefficient processing impairs release of RNA from the site of transcription. EMBO J. 18: $2855-2866$.

Farmerie, W.G. and Folk, W.R. 1984. Regulation of polyomavirus transcription by large tumor antigen. Proc. Natl. Acad. Sci. 81: 6919-6923.

Griffin, B. and Fried, M. 1975. Amplification of a specific region of the polyoma virus genome. Nature 256: 175-179.

$\mathrm{Gu}$, R., Zhang, Z., and Carmichael, G.G. 2006. How a small DNA virus uses dsRNA but not RNAi to regulate its life cycle. Cold Spring Harb. Symp. Quant. Biol. 71: 293-299.

Heiser, W.C. and Eckhart, W. 1982. Polyoma virus early and late mRNAs in productively infected mouse 3T6 cells. J. Virol. 44: 175188.

Hyde-DeRuyscher, R. and Carmichael, G.G. 1988. Polyomavirus early-late switch is not regulated at the level of transcription 
initiation and is associated with changes in RNA processing. Proc. Natl. Acad. Sci. 85: 8993-8997.

Hyde-DeRuyscher, R.P. and Carmichael, G.G. 1990. Polyomavirus late pre-mRNA processing: DNA replication-associated changes in leader exon multiplicity suggest a role for leader-to-leader splicing in the early-late switch. J. Virol. 64: 5823-5832.

Kamen, R., Lindstrom, D.M., Shure, H., and Old, R.W. 1974. Virusspecific RNA in cells productively infected or transformed by polyoma virus. Cold Spring Harb. Symp. Quant. Biol. 39: 187-198.

Kamen, R., Favaloro, J., and Parker, J. 1980a. Topography of the three late mRNA's of polyoma virus which encode the virion proteins. J. Virol. 33: 637-651.

Kamen, R., Favaloro, J., Parker, J., Treisman, R., Lania, L., Fried, M., and Mellor, A. 1980b. Comparison of polyoma virus transcription in productively infected mouse cells and transformed rodent cell lines. Cold Spring Harb. Symp. Quant. Biol. 44: 63-75.

Kamen, R., Jat, P., Treisman, R., Favaloro, J., and Folk, W.R. 1982. 5' Termini of polyoma virus early region transcripts synthesized in vivo by wild-type virus and viable deletion mutants. J. Mol. Biol. 159: 189-224.

Kaneko, S., Rozenblatt-Rosen, O., Meyerson, M., and Manley, J.L. 2007. The multifunctional protein $\mathrm{p} 54^{\mathrm{nrb}} / \mathrm{PSF}$ recruits the exonuclease XRN2 to facilitate pre-mRNA $3^{\prime}$ processing and transcription termination. Genes \& Dev. 21: 1779-1789.

Kim, S.J. and Martinson, H.G. 2003. Poly(A)-dependent transcription termination: continued communication of the poly(A) signal with the polymerase is required long after extrusion in vivo. J. Biol. Chem. 278: 41691-41701.

Kumar, M. and Carmichael, G.G. 1997. Nuclear antisense RNA induces extensive adenosine modifications and nuclear retention of target transcripts. Proc. Natl. Acad. Sci. 94: 3542-3547.

Liu, Z. and Carmichael, G.G. 1993. Polyoma virus early-late switch: Regulation of late RNA accumulation by DNA replication. Proc. Natl. Acad. Sci. 90: 8494-8498.

Liu, Z., Batt, D.B., and Carmichael, G.G. 1994. Targeted nuclear antisense RNA mimics natural antisense-induced degradation of polyoma virus early RNA. Proc. Natl. Acad. Sci. 91: 4258-4262.

Maquat, L.E. and Carmichael, G.G. 2001. Quality control of mRNA function. Cell 104: 173-176.

Peterson, J.A. and Myers, A.M. 1993. Functional analysis of mRNA 3' end formation signals in the convergent and overlapping transcription units of the S. cerevisiae genes RHO1 and MRP2. Nucleic Acids Res. 21: 5500-5508.
Piper, P. 1979. Polyoma virus transcription early during productive infection of mouse 3T6 cells. J. Mol. Biol. 131: 399-407.

Prescott, E.M. and Proudfoot, N.J. 2002. Transcriptional collision between convergent genes in budding yeast. Proc. Natl. Acad. Sci. 99: 8796-8801.

Puig, S., Perez-Ortin, J.E., and Matallana, E. 1999. Transcriptional and structural study of a region of two convergent overlapping yeast genes. Curr. Microbiol. 39: 369-373.

Ruley, E. and Fried, M. 1983. Sequence repeats in a polyoma virus DNA region important for gene expression. J. Virol. 47: 233-237.

Seo, G.J., Fink, L.H., O'Hara, B., Atwood, W.J., and Sullivan, C.S. 2008. Evolutionarily conserved function of a viral microRNA. J. Virol. 82: 9823-9828.

Seo, G.J., Chen, C.J., and Sullivan, C.S. 2009. Merkel cell polyomavirus encodes a microRNA with the ability to autoregulate viral gene expression. Virology 383: 183-187.

Sullivan, C.S., Grundhoff, A.T., Tevethia, S., Pipas, J.M., and Ganem, D. 2005. SV40-encoded microRNAs regulate viral gene expression and reduce susceptibility to cytotoxic T cells. Nature 435: 682-686.

Sullivan, C.S., Sung, C.K., Pack, C.D., Grundhoff, A., Lukacher, A.E., Benjamin, T.L., and Ganem, D. 2009. Murine polyomavirus encodes a microRNA that cleaves early RNA transcripts but is not essential for experimental infection. Virology. doi: 10.1016/ j.virol.2009.02.017.

Sun, M., Hurst, L.D., Carmichael, G.G., and Chen, J. 2005. Evidence for a preferential targeting of $3^{\prime}$-UTRs by cis-encoded natural antisense transcripts. Nucleic Acids Res. 33: 5533-5543.

Treisman, R. 1980. Characterization of polyoma late mRNA leader sequences by molecular cloning and DNA sequence analysis. Nucleic Acids Res. 8: 4867-4888.

Treisman, R. and Kamen, R. 1981. Structure of polyoma virus late nuclear RNA. J. Mol. Biol. 148: 273-301.

Wang, Q. and Carmichael, G.G. 2004. Effects of length and location on the cellular response to double-stranded RNA. Microbiol. Mol. Biol. Rev. 68: 432-452.

Wang, Q., Zhang, Z., Blackwell, K., and Carmichael, G.G. 2005. Vigilins bind to promiscuously A-to-I-edited RNAs and are involved in the formation of heterochromatin. Curr. Biol. 15: 384-391.

Zhang, Z. and Carmichael, G.G. 2001. The fate of dsRNA in the nucleus. A p54 ${ }^{\mathrm{nrb}}$-containing complex mediates the nuclear retention of promiscuously A-to-I edited RNAs. Cell 106: 465-475. 

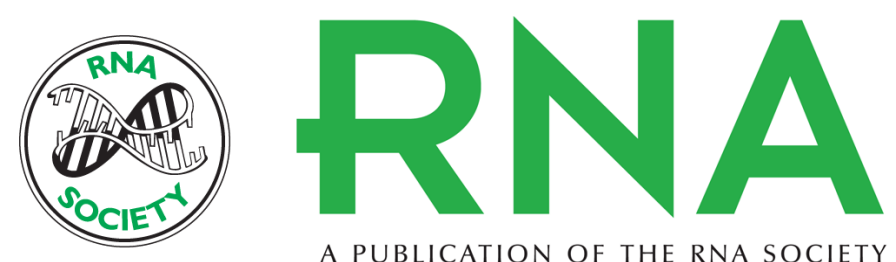

A PUBLICATION OF THE RNA SOCIETY

\section{Gene regulation by sense-antisense overlap of polyadenylation signals}

Rui Gu, Zuo Zhang, Joshua N. DeCerbo, et al.

RNA 2009 15: 1154-1163 originally published online April 23, 2009

Access the most recent version at doi:10.1261/rna.1608909

\section{Supplemental http://rnajournal.cshlp.org/content/suppl/2009/04/27/rna.1608909.DC1 \\ Material}

References This article cites 43 articles, 25 of which can be accessed free at: http://rnajournal.cshlp.org/content/15/6/1154.full.html\#ref-list-1

\section{License}

Email Alerting Receive free email alerts when new articles cite this article - sign up in the box at the Service top right corner of the article or click here. 